\title{
Flavour physics constraints in the BMSSM
}

\author{
N. Bernal, ${ }^{a}$ M. Losada $^{b}$ and F. Mahmoudi ${ }^{c, 1}$ \\ ${ }^{a}$ Physikalisches Institut and Bethe Center for Theoretical Physics, Universität Bonn, \\ Nußallee 12, D53115 Bonn, Germany \\ ${ }^{b}$ Centro de Investigaciones, Cra 3 Este No 47A-15, \\ Universidad Antonio Nariño, Bogotá, Colombia \\ ${ }^{c}$ CERN Theory Division, Physics Department, \\ CH-1211 Geneva 23, Switzerland \\ E-mail: nicolas@th.physik.uni-bonn.de, malosada@uan.edu.co, \\ mahmoudi@in2p3.fr
}

ABSTRaCt: We study the implications of the presence of the two leading-order, nonrenormalizable operators in the Higgs sector of the MSSM to flavour physics observables. We identify the constraints of flavour physics on the parameters of the BMSSM when we: a) focus on a region of parameters for which electroweak baryogenesis is feasible, b) use a CMSSM-like parametrization, and c) consider the case of a generic NUHM-type model. We find significant differences as compared to the standard MSSM case.

Keywords: Extended Supersymmetry, Rare Decays, Higgs Physics

ARXIV EPRINT: 1104.5395

\footnotetext{
${ }^{1}$ On leave from Clermont Université, Université Blaise Pascal, CNRS/IN2P3, LPC, BP 10448, 63000 Clermont-Ferrand, France.
} 


\section{Contents}

1 Introduction 1

2 The Higgs boson sector 2

2.1 The spectrum 2

2.2 The vacuum 4

3 Flavour constraints in the MSSM 4

$\begin{array}{lll}4 & \text { EWBG in the BMSSM framework } & 7\end{array}$

5 Results $\quad \mathbf{8}$

5.1 Flavour constraints on EWBG 9

$\begin{array}{lll}5.2 & \text { Flavour constraints for a CMSSM-like model } & 12\end{array}$

$\begin{array}{lll}5.3 & \text { Flavour constraints for a NUHM-like model } & 13\end{array}$

6 Conclusions 16

\section{Introduction}

The Minimal Supersymmetric Standard Model (MSSM) is one of the most studied models that probes effects from beyond the Standard Model (SM) physics. Although the MSSM can provide many interesting features to physics beyond the SM, there are severe constraints on the parameters when trying to provide a feasible scenario for a Higgs boson mass consistent with experimental constraints, electroweak baryogenesis (EWBG), dark matter, and flavour physics observables.

Within the MSSM, the tree level bound on the lightest Higgs mass is violated, and hence significant corrections arising from loops of top quarks and squarks are necessary. But in order for these effects to account for the Higgs mass, the top squarks must be quite massive or the top squarks must be highly mixed. This suggests that if low energy supersymmetry is important to the solution of the hierarchy problem, there are likely to be additional degrees of freedom in the theory beyond those of the MSSM. Recently there has been a great interest in extensions of the MSSM by higher-dimension operators [1-12]. These may have an important impact on the Higgs sector, alleviating in particular the tension in the MSSM that results from the LEP Higgs bounds, i.e. the so-called little hierarchy problem. In particular, an attractive extension of the MSSM is the "Beyond the MSSM" (BMSSM) scenario [6]. Here, in addition to the MSSM superpotential a nonrenormalizable contribution to the Higgs sector is included

$$
W_{\mathrm{BMSSM}}=W_{\mathrm{MSSM}}+\frac{\lambda}{M}\left(H_{u} H_{d}\right)^{2},
$$


as well as a contribution to the soft SUSY-breaking Lagrangian

$$
\mathcal{L}_{\text {soft }}^{\mathrm{BMSM}}=\mathcal{L}_{\mathrm{soft}}^{\mathrm{MSSM}}+\frac{\lambda_{s} m_{\mathrm{SUSY}}}{M}\left(H_{u} H_{d}\right)^{2},
$$

where $M$ and $m_{\text {SUSY }}$ are the energy scale of new physics and the energy scale of SUSY breaking, respectively. The new terms in the previous equations account for the leading supersymmetric and $F$-term supersymmetry breaking corrections to the Higgs sector from a new threshold at mass scale $M$ [1-12]. The possible ultraviolet completions can vary significantly, being the simplest the addition of a singlet scalar field, as in the case of the NMSSM $[13,14]$. It is worth noticing that with the parametrization of eqs. (1.1) and (1.2), we can capture the main effects in the effective theory allowing a model-independent description of a large class of extensions of the MSSM, irrespective of the specific UV completion. To parametrize the corrections that modify the spectrum and interactions we use the dimensionless parameters

$$
\epsilon_{1} \equiv \frac{\lambda \mu^{*}}{M}, \quad \epsilon_{2} \equiv-\frac{\lambda_{s} m_{\mathrm{SUSY}}}{M} .
$$

For $M$ of the order of a few $\mathrm{TeV}$, the non-renormalizable corrections of the BMSSM are sizeable and they have been analyzed in the context of electroweak baryogenesis [15-17], dark matter [16, 18-24], Higgs phenomenology [25-27] and collider phenomenology [28, 29]. In a nutshell, it has been shown that the consequence of this simple extension of the MSSM including higher order operators which are suppressed by inverse powers of a scale of new physics somewhat higher than the electroweak breaking scale, can significantly relax the constraints coming from the above mentioned physical observables.

In this work, we focus on the implications of the BMSSM framework when considering flavour physics observables. We do this in particular for three specific scenarios: a generic choice of BMSSM parameters for which EWBG is viable, and two general frameworks inspired by models where the supersymmetric parameters are correlated such as the constrained supersymmetric extension of the Standard Model (CMSSM) scenario [30-33] and models with non-universal Higgs masses (NUHM) [34].

In the next section we briefly recall the main features of the model in the Higgs sector. Section 3 summarizes the main flavour observables and their current experimental values that we will use in our analysis. In section 4 we briefly present the main effects from having a viable electroweak baryogenesis scenario in the BMSSM, which defines the interesting region in parameter space. Finally in section 5 we present the main results of this work which describes the constraints from flavour observables for the different parametrizations of the BMSSM that we have considered: the region for which EWBG is viable and two frameworks inspired by the CMSSM and the NUHM models.

\section{The Higgs boson sector}

\subsection{The spectrum}

We define the scalar Higgs components by

$$
H_{d}=\left(\begin{array}{c}
H_{d}^{0} \\
H_{d}^{-}
\end{array}\right)=\left(\begin{array}{c}
\frac{\phi_{1}+H_{d r}+i H_{d i}}{\sqrt{2}} \\
H_{d}^{-}
\end{array}\right) \quad H_{u}=\left(\begin{array}{c}
H_{u}^{+} \\
H_{u}^{0}
\end{array}\right)=\left(\begin{array}{c}
H_{u}^{+} \\
\frac{\phi_{2}+H_{u r}+i H_{u i}}{\sqrt{2}}
\end{array}\right) .
$$


The vacuum expectation values of these Higgs fields are parameterized by

$$
\begin{aligned}
\left\langle H_{d}^{0}\right\rangle & =\phi_{1} / \sqrt{2}, & \left\langle H_{u}^{0}\right\rangle & =\phi_{2} / \sqrt{2}, \\
\tan \beta & =\left|\phi_{2} / \phi_{1}\right|, & v & =\sqrt{\left(\phi_{1}^{2}+\phi_{2}^{2}\right) / 2} \simeq 174 \mathrm{GeV} .
\end{aligned}
$$

To leading order, the two charged and four neutral Higgs mass eigenstates are related to the interaction eigenstates via

$$
\begin{aligned}
\left(\begin{array}{c}
H_{d}^{*+} \\
H_{u}^{+}
\end{array}\right) & =\left(\begin{array}{cc}
s_{\beta} & -c_{\beta} \\
c_{\beta} & s_{\beta}
\end{array}\right)\left(\begin{array}{c}
H^{+} \\
G^{+}
\end{array}\right), \\
\left(\begin{array}{c}
H_{d i} \\
H_{u i}
\end{array}\right) & =\left(\begin{array}{cc}
s_{\beta} & -c_{\beta} \\
c_{\beta} & s_{\beta}
\end{array}\right)\left(\begin{array}{c}
A \\
G^{0}
\end{array}\right), \\
\left(\begin{array}{l}
H_{d r} \\
H_{u r}
\end{array}\right) & =\left(\begin{array}{cc}
c_{\alpha} & -s_{\alpha} \\
s_{\alpha} & c_{\alpha}
\end{array}\right)\left(\begin{array}{c}
H \\
h
\end{array}\right),
\end{aligned}
$$

where $c_{\beta} \equiv \cos \beta, s_{\beta} \equiv \sin \beta$, and similarly for $\alpha$. Within the MSSM (without the $\epsilon_{i}$ operators), the angle $\alpha$ is given (at tree level) by

$$
s_{2 \alpha}=-\frac{m_{A}^{2}+m_{Z}^{2}}{m_{H}^{2}-m_{h}^{2}} s_{2 \beta} .
$$

If the $\epsilon_{1,2}$ couplings are complex, then the four neutral mass eigenstates are related by a $4 \times 4$ transformation matrix to the real and imaginary components of $H_{d}^{0}$ and $H_{u}^{0}$. In the unitary gauge, the Goldstone fields $G^{ \pm}$and $G^{0}$ are set to zero.

The main effects of the non-renormalizable operators of eqs. (1.1) and (1.2) appear on the Higgs masses. Taking the $Z$ boson mass $m_{Z}$, the pseudoscalar Higgs boson mass $m_{A}$ and the ratio of the two vacuum expectation values $\tan \beta$ as input parameters, we obtain the leading order corrections in $\epsilon_{i}$ to the Higgs spectrum:

$$
\begin{aligned}
\delta_{\epsilon} m_{h}^{2} & =2 v^{2}\left(\epsilon_{2 r}-2 \epsilon_{1 r} s_{2 \beta}-\frac{2 \epsilon_{1 r}\left(m_{A}^{2}+m_{Z}^{2}\right) s_{2 \beta}+\epsilon_{2 r}\left(m_{A}^{2}-m_{Z}^{2}\right) c_{2 \beta}^{2}}{\sqrt{\left(m_{A}^{2}-m_{Z}^{2}\right)^{2}+4 m_{A}^{2} m_{Z}^{2} s_{2 \beta}^{2}}}\right), \\
\delta_{\epsilon} m_{H}^{2} & =2 v^{2}\left(\epsilon_{2 r}-2 \epsilon_{1 r} s_{2 \beta}+\frac{2 \epsilon_{1 r}\left(m_{A}^{2}+m_{Z}^{2}\right) s_{2 \beta}+\epsilon_{2 r}\left(m_{A}^{2}-m_{Z}^{2}\right) c_{2 \beta}^{2}}{\sqrt{\left(m_{A}^{2}-m_{Z}^{2}\right)^{2}+4 m_{A}^{2} m_{Z}^{2} s_{2 \beta}^{2}}}\right), \\
\delta_{\epsilon} m_{H^{ \pm}}^{2} & =2 v^{2} \epsilon_{2 r}
\end{aligned}
$$

where $\epsilon_{1 r}$ and $\epsilon_{2 r}$ are the real parts of $\epsilon_{1}$ and $\epsilon_{2}$.

The mixing angle $\alpha$ is shifted from its MSSM value:

$$
\begin{aligned}
s_{2 \alpha} & =\frac{-\left(m_{A}^{2}+m_{Z}^{2}\right) s_{2 \beta}+4 v^{2} \epsilon_{1 r}}{\left(m_{H}^{2}-m_{h}^{2}\right) s_{2 \beta}} \\
& =-\frac{\left(m_{A}^{2}+m_{Z}^{2}\right) s_{2 \beta}}{\left(m_{A}^{4}-2 m_{A}^{2} m_{Z}^{2} c_{4 \beta}+m_{Z}^{4}\right)^{1 / 2}}-4 v^{2} c_{2 \beta}^{2} \frac{2 \epsilon_{1 r}\left(m_{A}^{2}-m_{Z}^{2}\right)^{2}-\epsilon_{2 r} s_{2 \beta}\left(m_{A}^{4}-m_{Z}^{4}\right)}{\left(m_{A}^{4}-2 m_{A}^{2} m_{Z}^{2} c_{4 \beta}+m_{Z}^{4}\right)^{3 / 2}} .
\end{aligned}
$$


In the MSSM, the Higgs boson mass can only be larger than the lower bound given by experimental constraints when large radiative corrections essentially arising from the stops are invoked: at least one of the stop mass eigenstates should be rather heavy and/or leftright-stop mixing should be substantial. On the other hand, in the BMSSM the additional operators contribute at tree-level to the lightest Higgs boson mass and diminish the tensions associated with the stop sector and $\tan \beta$. Now, the bound on the Higgs boson mass allows for stops that are relatively light and unmixed.

\subsection{The vacuum}

Previous work has shown that in a generic extension of the MSSM with additional nonrenormalizable interactions two types of vacua can exist, the MSSM-like vacua and the 'supersymmetric electroweak symmetry breaking' (sEWSB) vacua [12]. There are significant differences amongst them and therefore the phenomenology both in the Higgs sector and other sectors of this supersymmetric model can vary considerably. Specifically for the present study we perform here we will restrict ourselves to the MSSM-like vacua and carefully check for the (meta)stability of the vacuum at each point in parameter space as we scan. To ensure this the couplings $\epsilon_{1}$ and $\epsilon_{2}$ cannot take on arbitrary values.

The inclusion of the BMSSM operators may destabilize the scalar potential. If $4\left|\epsilon_{1}\right|>$ $\epsilon_{2}$, the effective quartic coupling along one of the D-flat directions is negative, causing a remote vacuum to form in the presence of which the electroweak MSSM-like vacuum could become metastable. We make the conservative assumption that when considering values of $\epsilon_{1} \gtrsim-0.1$, vacuum stability is ensured provided that the following condition is fulfilled [35]:

$$
\frac{m_{A}^{2}(1+\sin 2 \beta)}{|\mu|^{2}} \geqslant 2\left(\frac{\tilde{\epsilon}}{\epsilon_{1}}\right)^{2}\left[1+\frac{m_{Z}^{2}}{m_{A}^{2}} \frac{16 \tilde{\epsilon}}{g_{Z}^{2}}\left(\frac{1+2 \sin 2 \beta}{1+\sin 2 \beta}-\frac{3}{2} \frac{\epsilon_{1}}{\tilde{\epsilon}}\right)\right]^{-1}
$$

where $\tilde{\epsilon}=\frac{1}{4} \epsilon_{2}+\epsilon_{1}$ and $g_{Z}^{2}=g^{2}+g^{\prime 2}$ with $g$ and $g^{\prime}$ the SM gauge couplings.

This will be the condition that we will apply below in the scans. We illustrate in figure 1 , the region in the $\tan \beta$ vs $m_{A}$ plane, having fixed the values of $\epsilon_{1}=-0.1$ and $\epsilon_{2}=0.05$ for which the MSSM-like vacuum is (meta)stable. The regions of the parameter space ruled out by the LEP bound on the Higgs mass are denoted in blue. Both the tree level contribution and the correction coming from the dimension-5 operators (c.f. eq. (2.5)) are taken into account. High values for $\tan \beta$ are usually excluded; only for values around $m_{A} \sim m_{Z}$, higher values for $\tan \beta \gtrsim 10$ are allowed. The orange lines correspond to contour levels for the vacuum constraint and for different values of the $\mu$ parameter: 110, 150, 250 and $350 \mathrm{GeV}$. For each value of $\mu$, the regions on the right hand side of the contour lines are excluded by the vacuum stability constraint described in eq. (2.9).

\section{Flavour constraints in the MSSM}

Flavour physics observables are very sensitive to new physics effects and can play an important role in disentangling different scenarios. In particular, they have been studied 


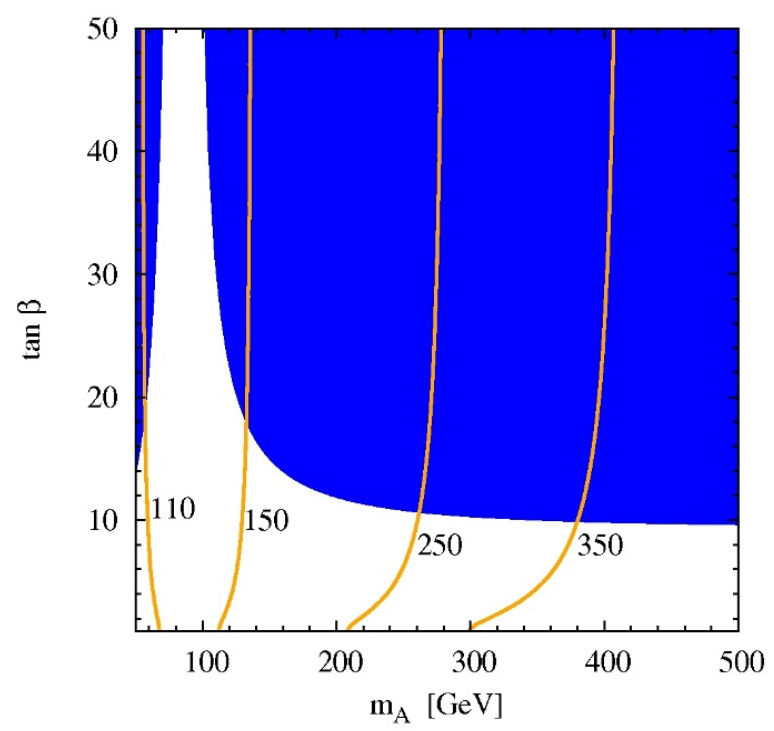

Figure 1. Regions in the $\left(m_{A}, \tan \beta\right)$ plane ruled out by the LEP bound on the Higgs mass (blue). The regions on the right hand side of the orange lines generate an unstable vacuum and are therefore excluded. These lines correspond to $\mu=110,150,250$ and $350 \mathrm{GeV} . \epsilon_{1}$ and $\epsilon_{2}$ are set to -0.1 and 0.05 respectively.

extensively and severe constraints have been obtained on the parameters of the MSSM [3638]. Here we compute the most constraining flavour observables with the SuperIso program [39, 40].

The transition which is most often discussed in this context is the flavour changing neutral current process $b \rightarrow s \gamma$ [41-43]. Since this transition occurs first at one-loop level in the SM, the new physics contributions can be of comparable magnitude.

The latest combined experimental value for this branching ratio is reported by the Heavy Flavor Averaging Group (HFAG) [44]:

$$
\operatorname{BR}\left(\bar{B} \rightarrow X_{s} \gamma\right)^{\exp }=(3.55 \pm 0.24 \pm 0.09) \times 10^{-4} .
$$

Following $[45,46]$, we calculate this branching ratio at the NNLO accuracy. With the most up-to-date parametric inputs as given in [47] the SM prediction reads: ${ }^{1}$

$$
\operatorname{BR}\left(\bar{B} \rightarrow X_{s} \gamma\right)^{\mathrm{SM}}=(3.06 \pm 0.22) \times 10^{-4} .
$$

The allowed range at 95\% C.L. for this branching ratio, including both the theoretical and experimental uncertainties is [40]:

$$
2.16 \times 10^{-4} \leq \mathrm{BR}\left(\bar{B} \rightarrow X_{s} \gamma\right) \leq 4.93 \times 10^{-4} .
$$

In contrast to the $b \rightarrow s \gamma$ transitions, the process $B_{u} \rightarrow \tau \nu_{\tau}$ is sensitive to the charged Higgs boson already at tree level. Since this decay is helicity suppressed in the SM, whereas there is no such suppression for the charged Higgs boson exchange, these two contributions

\footnotetext{
${ }^{1}$ The slight difference compared to earlier published results is explained by the parametric updates.
} 
can be of similar magnitude in the limit of high $\tan \beta[48,49]$. This decay is thus very sensitive to the charged Higgs boson and provides important constraints.

The current HFAG value for $\operatorname{BR}\left(B_{u} \rightarrow \tau \nu_{\tau}\right)$ is [44]

$$
\mathrm{BR}\left(B_{u} \rightarrow \tau \nu_{\tau}\right)^{\exp }=(1.64 \pm 0.34) \times 10^{-4} .
$$

The evaluation of $\operatorname{BR}\left(B_{u} \rightarrow \tau \nu_{\tau}\right)$ suffers however from the uncertainties in the determination of $\left|V_{u b}\right|$. We consider the following ratio to express the new physics contributions:

$$
R_{\tau \nu_{\tau}}=\frac{\mathrm{BR}\left(B_{u} \rightarrow \tau \nu_{\tau}\right)^{\mathrm{NP}}}{\mathrm{BR}\left(B_{u} \rightarrow \tau \nu_{\tau}\right)^{\mathrm{SM}}}=\left[1-\left(\frac{m_{B}^{2}}{M_{H^{+}}^{2}}\right) \frac{\tan ^{2} \beta}{1+\epsilon_{0} \tan \beta}\right]^{2}
$$

In the $\mathrm{SM}, R_{\tau \nu_{\tau}}^{\mathrm{SM}}=1$. The experimental result for this ratio is [44]:

$$
R_{\tau \nu_{\tau}}^{\exp }=1.63 \pm 0.54
$$

leading to the following allowed interval at $95 \%$ C.L. [40]:

$$
0.56<R_{\tau \nu_{\tau}}<2.70
$$

The semileptonic decays $B \rightarrow D \ell \nu$ [50-52] have the advantage of depending on $\left|V_{c b}\right|$, which is known to better precision than $\left|V_{u b}\right|$. In addition, the $\operatorname{BR}\left(B \rightarrow D \tau \nu_{\tau}\right)$ is about 50 times larger than $\operatorname{BR}\left(B_{u} \rightarrow \tau \nu_{\tau}\right)$ in the SM. Due to the presence of at least two neutrinos in the final state, the experimental determination remains however very complex. To reduce some of the theoretical uncertainties, we consider the following ratio [52]:

$$
\xi_{D \ell \nu} \equiv \frac{\mathrm{BR}\left(B \rightarrow D \tau \nu_{\tau}\right)}{\mathrm{BR}\left(B \rightarrow D e \nu_{e}\right)} .
$$

The SM prediction for this ratio is [40]

$$
\xi_{D \ell \nu}^{\mathrm{SM}}=(29 \pm 3) \times 10^{-2},
$$

and the experimental result by the BaBar collaboration reads [53]

$$
\xi_{D \ell \nu}^{\exp }=(41.6 \pm 11.7 \pm 5.2) \times 10^{-2} .
$$

The $95 \%$ C.L. allowed interval is given by [40]

$$
0.151<\xi_{D \ell \nu}<0.681 \text {. }
$$

In analogy to the case for $B_{u} \rightarrow \tau \nu_{\tau}$, charged Higgs bosons would also contribute to the decays $D_{s} \rightarrow \tau \nu_{\tau}$ at tree level $[48,54-57]$. The experimental results for this branching ratio is $[44,57]$ :

$$
\operatorname{BR}\left(D_{s} \rightarrow \tau \nu_{\tau}\right)^{\exp }=(5.38 \pm 0.32) \times 10^{-2},
$$

while the SM prediction reads:

$$
\operatorname{BR}\left(D_{s} \rightarrow \tau \nu_{\tau}\right)^{\mathrm{SM}}=(5.10 \pm 0.13) \times 10^{-2},
$$


in which $f_{D_{s}}=248 \pm 2.5 \mathrm{MeV}$ [58] is used. We consider the following allowed interval at 95\% C.L.:

$$
4.7 \times 10^{-2}<\operatorname{BR}\left(D_{s} \rightarrow \tau \nu_{\tau}\right)<6.1 \times 10^{-2} .
$$

The last leptonic decay that we consider is the decay $K \rightarrow \mu \nu_{\mu}$, and in particular we consider the ratio [59]

$$
R_{\ell 23}=\left|1-\frac{m_{K^{+}}^{2}}{M_{H^{+}}^{2}}\left(1-\frac{m_{d}}{m_{s}}\right) \frac{\tan ^{2} \beta}{1+\epsilon_{0} \tan \beta}\right| .
$$

The SM prediction for this ratio is $R_{\ell 23}^{\mathrm{SM}}=1$ and the experimental measurement gives:

$$
R_{\ell 23}^{\exp }=1.004 \pm 0.007,
$$

where $f_{K} / f_{\pi}=1.189 \pm 0.007$ is used [60], and the allowed interval at $95 \%$ C.L. read:

$$
0.990<R_{\ell 23}<1.018 .
$$

In the following sections, we study the regions excluded in the BMSSM parameter space by the flavour observables.

\section{EWBG in the BMSSM framework}

Electroweak baryogenesis (EWBG) is an attractive mechanism for generating the baryon asymmetry of the Universe (BAU), in part due to its testability. However, for this mechanism to be successful in the MSSM the parameter space is reduced to a finely tuned region.

In the stop sector of the MSSM a strong first-order phase transition requires at least one light stop (which must be mostly right-handed $\left(\widetilde{t}_{R}\right)$, to avoid large contributions to the $\rho$ parameter and due to null searches for a light sbottom [47, 61]). At the same time, the large radiative corrections needed to increase the Higgs boson mass above the LEP bound $m_{h}>114 \mathrm{GeV}$ [47] require that at least one stop $\left(\widetilde{t}_{L}\right)$ is very heavy [62]. The electroweak phase transition was studied in an effective theory with a large stop hierarchy, concluding that successful EWBG is possible only for $m_{\tilde{t}_{R}}<125 \mathrm{GeV}$ and $m_{\tilde{t}_{L}}>6.5 \mathrm{TeV}$ [63], making the scenario finely tuned.

More recently, it was shown $[15,16]$ that in the BMSSM the left-handed stop can also be relatively light, providing additional bosonic degrees of freedom that strengthen the first-order phase transition. The $\rho$ parameter and direct searches for a light sbottom are now the main constraint.

On the other hand, large values of the pseudo-scalar Higgs boson, $m_{A}$, are preferred (i) to make the electroweak phase transition [63-82] more strongly first-order, and (ii) to evade constraints from $b \rightarrow s \gamma$ [83]. However, the production of left-handed charge during EWBG is enhanced when $m_{A}$ is light.

In the BMSSM, the pseudoscalar Higgs boson can be significantly lighter as the phase transition is strengthened by having lighter stops.

The value of $\tan \beta$ is limited from a compromise in giving a large enough value of the Higgs mass versus a strong enough phase transition, and in contrast from the constraints from $b \rightarrow s \gamma$ for small values of $m_{A}$. 
As far as the EWBG in the BMSSM, given that the Higgs boson mass is made large enough from the additional corrections from the non-renormalizable operators, $\tan \beta$ can take on smaller values. Let us recall that large values for $\tan \beta$ decrease the contribution to the Higgs boson mass coming from the dimension 5 operators, and in general values of $\tan \beta \gtrsim 10$ tend to be unfavourable. However, for $m_{A} \sim m_{Z}$ the uplift of the Higgs boson mass is maximal and therefore $\tan \beta$ could take much higher values.

\section{CP violation for BAU production}

In the MSSM, the CP-violating phases that drive EWBG arise in the gaugino/higgsino sector and at the same time contribute to electric dipole moments (EDMs). One-loop contributions can be sufficiently suppressed by making the first two squark and slepton generations heavy, however, there exist two-loop contributions that cannot be suppressed without spoiling EWBG and give a minimum value of the EDM. The main conclusion is that EWBG with universal gaugino phases is nearly ruled out. With improvements by a factor 3-4 in the upper bounds on the EDMs of the electron or the neutron, MSSM baryogenesis will be possible only in the so-called "bino-driven" scenario, where the CPviolating phase associated with the $\mathrm{U}(1)_{Y}$ gaugino is tuned to be much larger than that of the $\mathrm{SU}(2)_{L}$ gaugino $[83,84]$.

In contrast in the BMSSM, new phases arise that can produce the baryon asymmetry of the Universe [17] and relax the constraints on MSSM parameters. In fact, the possibility of spontaneous baryogenesis determined from the phase in the Higgs sector must be taken into account. Furthermore, the top and stop CP-violating sources are now unsuppressed in comparison to the MSSM case. In fact the MSSM phases can be zero and still EWBG is viable, however, if the experimental sensitivity of electric dipole moment experiments is increased by one order of magnitude in the BMSSM scenario an EDM signal should be detected.

The imaginary parts of $\epsilon_{1}$ and $\epsilon_{2}$ are constrained from EDMs [17]. However, we will for simplicity take them to be zero in this work, otherwise the Higgs scalar sector is significantly affected and a more complicated scenario arises.

\section{Results}

For the numerical evaluation we use SuperIso v2.8 [39, 40], and the spectra of SUSY particles are calculated using a modified version of SuSpect $[16,85]$ incorporating the leading order corrections in $\epsilon_{i}$ to the Higgs masses and to the mixing angle $\alpha$. The top quark pole mass is set to $m_{t}=173.3 \mathrm{GeV}$ [86].

For each observable we determine the regions excluded in the BMSSM parameter space. We perform the scans in the following way: we first impose the vacuum stability constraint, the constraint on the value of the lightest Higgs boson mass, the constraint from fulfilling the $b \rightarrow s \gamma$ branching ratio, followed by the rest of the flavour constraints.

To understand correctly the plots below, it is important to keep in mind that we are performing a multiple-parameter scan, as such when projected onto a two-dimensional plane all other parameters can take on many different values. 


\subsection{Flavour constraints on EWBG}

We present our first results in the case in which we fix the parameters in the BMSSM such that the electroweak baryogenesis scenario is feasible. As mentioned above we do not consider in this work the effect of complex values for $\epsilon_{1}$ and $\epsilon_{2}$. Based on the discussion presented in previous sections we fix the parameters of the effective theory as follows: we take the gaugino masses to be $M_{1}=100 \mathrm{GeV}, M_{2}=200 \mathrm{GeV}$ and $M_{3}=1000 \mathrm{GeV}$. The right handed stop $m_{\tilde{t}_{R}}=160 \mathrm{GeV}$, for the left-handed supersymmetry breaking stop mass we scan in the range $150<m_{Q_{3}}<300 \mathrm{GeV}$. The Higgsino mass parameter $100<$ $\mu<400 \mathrm{GeV}$. We assume positive values for the $\mu$ parameter since this makes it easier to explain $(g-2)_{\mu}$ data and to satisfy $b \rightarrow s \gamma$ constraints. The trilinear scalar couplings $0<A_{t}=A_{c}=A_{u}<200 \mathrm{GeV}$. The other sleptons and squarks soft masses are fixed to $m_{Q}=m_{L}=1000 \mathrm{GeV}$. We generated randomly over 400,000 points satisfying the above requirements.

Recall that the flavour observables that we focus on are essentially: $b \rightarrow s \gamma, B \rightarrow \tau \nu$, $R_{l 23}, B \rightarrow D \ell \nu$ and $D_{s} \rightarrow \tau \nu$.

In figure 2 we compare the constraints in the $\tan \beta$ vs $m_{H^{+}}$plane with (lower panels) and without (upper panel) the terms arising from the higher dimensional operators. In the plots, in addition to scanning over the SUSY parameters as described above, we scan over values of $-0.1<\epsilon_{1}<0$ and $0<\epsilon_{2}<0.1$ (central panel) or $-0.1<\epsilon_{2}<0$ (lower panel). The flavour constraints are applied in the order given in the legend after the vacuum stability and lightest Higgs mass limits: the points excluded by $b \rightarrow s \gamma$ are shown in red. On top of that are displayed consecutively the points excluded by $B \rightarrow \tau \nu$ (in blue), $R_{l 23}$ (in magenta), $B \rightarrow D \ell \nu$ (in orange) and $D_{s} \rightarrow \tau \nu$ (in grey). The points satisfying all the constraints (allowed) are displayed at the end in green on top of all the other colours. It is clear that different flavour observables rule out some of the regions and we observe that a wide band, in the range of $\tan \beta \lesssim 15$ for $\epsilon_{2}>0$ and $\tan \beta \lesssim 10$ for $\epsilon_{2}<0$, for $m_{H^{+}} \gtrsim 150-200 \mathrm{GeV}$, that was excluded in the MSSM, can now satisfy all constraints. This occurs given our choices for the values of the stop masses, which in the MSSM need to be very large for the Higgs boson to avoid the experimental limit. In the BMSSM this can be avoided as mentioned above and thus the region would be allowed from flavour physics constraints. There are also some scattered allowed points for $30<\tan \beta<40$ when $m_{H^{+}}>300 \mathrm{GeV}$. It is important to note that the lowest excluded values of $\tan \beta$, as a function of the charged Higgs boson mass, from the $b \rightarrow s \gamma$ constraint are increased in the BMSSM case as compared to the MSSM, with the constraint being stronger in the case of $\epsilon_{2}<0$.

The vacuum stability and the lightest Higgs boson mass constraint are not visible since they are displayed in the background. However, as expected from equation (2.9), the vacuum constraint tends to be stronger for low values of the charged Higgs mass (implying low $m_{A}$ values) and for negative values for $\epsilon_{2}$, and the region with $m_{H^{+}} \lesssim 200 \mathrm{GeV}$ becomes excluded. In total, about $8 \%$ of the generated points satisfy all the constraints in the case of $\epsilon_{2}>0$ while $5 \%$ of the points survive for $\epsilon_{2}<0$.

We conclude that for the EWBG scenario only the regions of low $\tan \beta$ and $m_{H^{+}} \gtrsim$ 

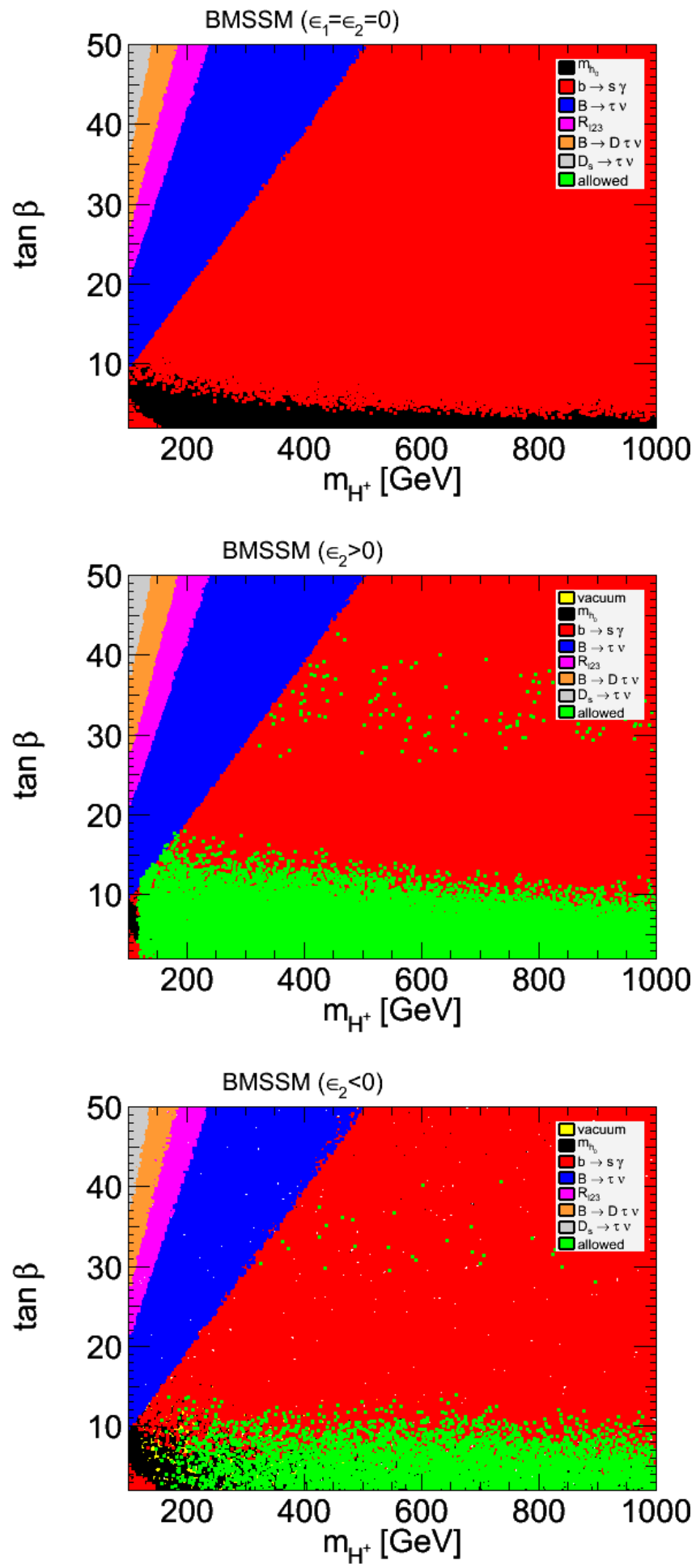

Figure 2. Flavour constraints on the $\left(m_{H^{+}}, \tan \beta\right)$ plane in the MSSM (upper) and the BMSSM (central and lower) for the scenario where EWBG is feasible, as described in text. The central panel corresponds to $\epsilon_{2}>0$ and the lower panel to $\epsilon_{2}<0$. 

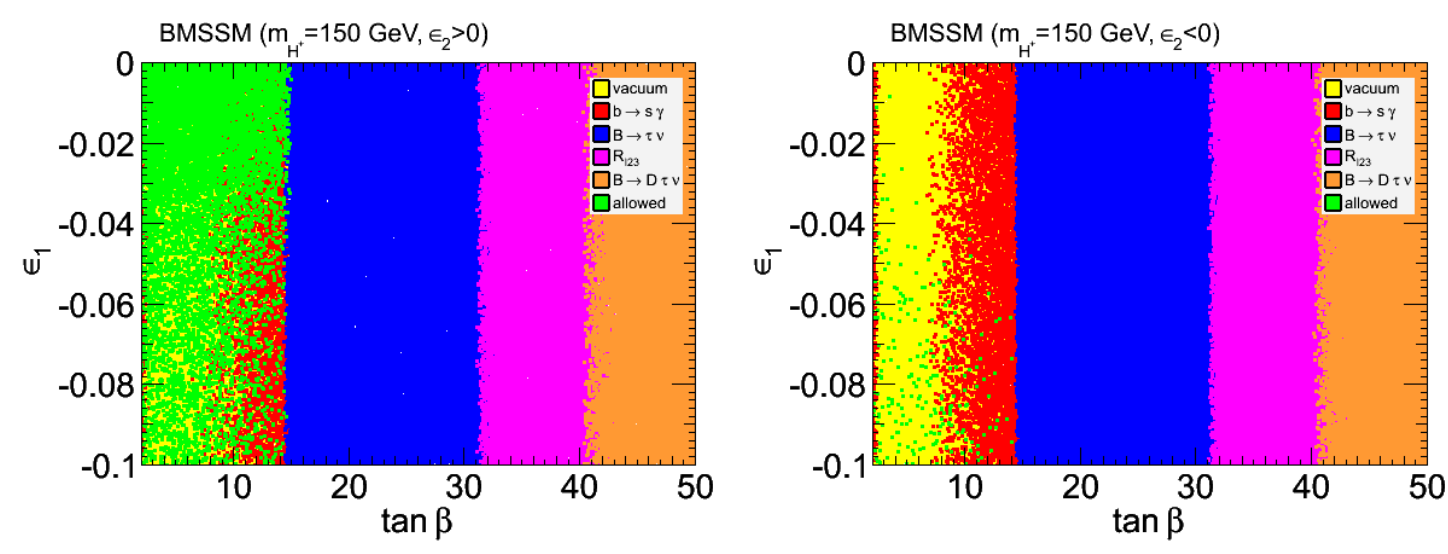

Figure 3. Flavour constraints on the $\left(\tan \beta, \epsilon_{1}\right)$ plane in the BMSSM for the scenario where EWBG is feasible. We used $m_{H^{ \pm}}=150 \mathrm{GeV}$ and $\epsilon_{2}$ positive (left panel) or $\epsilon_{2}$ negative (right panel).

$150-200 \mathrm{GeV}$ and a few points above $\tan \beta=25$ and $m_{H^{+}} \lesssim 300 \mathrm{GeV}$ will remain viable.

In figure 3 we show the regions of parameter space that are allowed after applying all constraints; in this case we are setting the charged Higgs boson mass $m_{H^{+}}=150 \mathrm{GeV}$. We do this separately for both $\epsilon_{2}>0$ (left panel) and $\epsilon_{2}<0$ (right panel).

In this figure, the flavour observables (and in particular $b \rightarrow s \gamma$ and $B \rightarrow \tau \nu_{\tau}$ ) are quite restrictive leaving the allowed region limited to values of $\tan \beta \lesssim 15$. Moreover, let us note that the vacuum stability constraint cuts out regions of parameter space that otherwise would be allowed purely from flavour constraints. In the case of $\epsilon_{2}<0$, the vacuum stability condition is especially constraining, limiting the allowed parameter space to values of $\epsilon_{1} \lesssim-0.05$. On the other hand, for $\epsilon_{2}>0$ the vacuum condition tends to be alleviated, and consequently the allowed region satisfying all constraints is defined over the full range of $\epsilon_{1}$, with a mild dependence on $\tan \beta$. The different flavour constraints have essentially the same effect for both cases with $\epsilon_{2}>0$ and $\epsilon_{2}<0$.

We next study in detail in figure 4 the constraining effect from the flavour observables and the vacuum stability constraint in the plane $\mu$ vs $\tan \beta$ for three different values of the charged Higgs boson mass: $m_{H^{+}}=150$ (upper panels), 250 (central panels) and $350 \mathrm{GeV}$ (lower panels); and for $\epsilon_{2}$ positive (left panels) and negative (right panels).

In the $\epsilon_{2}>0$ case with the increase of $m_{H^{ \pm}}$the allowed parameter space tends to shrink mainly due to the strengthening of the $b \rightarrow s \gamma$ constraint for low values of the $\mu$ parameter. On the contrary, the bounds given by all the other flavour observables and in particular by $B \rightarrow \tau \nu_{\tau}$ weaken. The vacuum condition is a very weak constraint for positive values of $\epsilon_{2}$. However, for negative values this condition is much stronger. In this case there is a significant effect in pushing the upper bound on $\mu$ to larger values, up to $\mu \sim 300 \mathrm{GeV}$ for $m_{H^{ \pm}}=350 \mathrm{GeV}$. For increasing $m_{H^{+}}$values, the constraint from $B \rightarrow \tau \nu_{\tau}$ also weakens but still eliminates regions in parameter space with larger values of $\tan \beta$. This is consistent with the fact mentioned above of the dependence at tree level of the $B \rightarrow \tau \nu_{\tau}$ with the charged Higgs boson. The constraint from $b \rightarrow s \gamma$ still limits the allowed range to $\tan \beta \lesssim 12$. Note also that some allowed points appear for $30<\tan \beta<35$, 

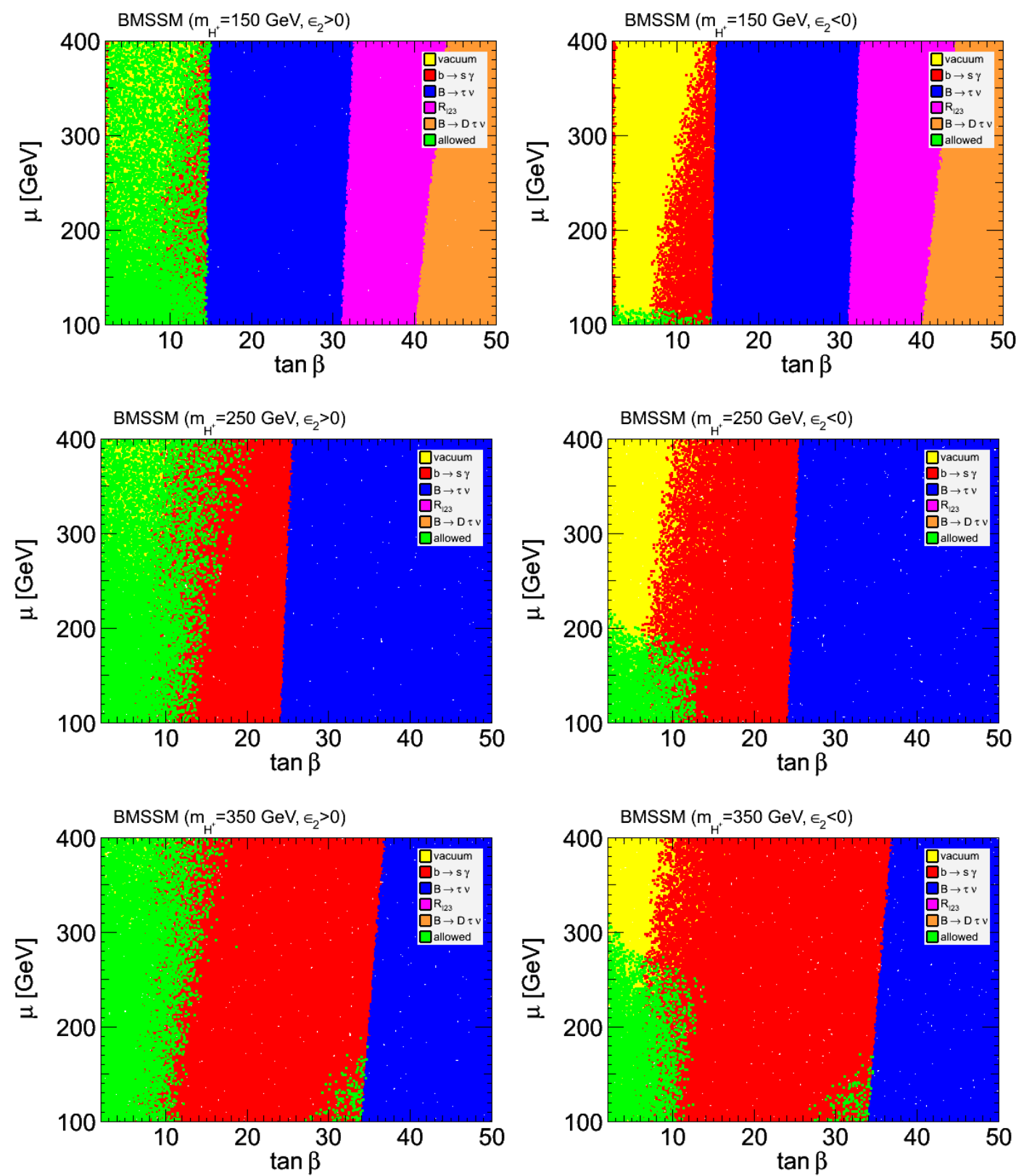

Figure 4. Flavour constraints on the $(\tan \beta, \mu)$ plane in the BMSSM for the scenario where EWBG is feasible. We used $\epsilon_{2}>0$ for the left panels and $\epsilon_{2}<0$ for the right panels. We also set $m_{H^{ \pm}}=150 \mathrm{GeV}$ (upper panels), $m_{H^{ \pm}}=250 \mathrm{GeV}$ (central panels) and $m_{H^{ \pm}}=350 \mathrm{GeV}$ (lower panels).

with $\mu \lesssim 200 \mathrm{GeV}$ when $m_{H^{+}}=350 \mathrm{GeV}$. Similar effects are also seen in the $\epsilon_{2}>0$ case. That is the role played by the constraint from $b \rightarrow s \gamma$ which is highlighted for intermediate values of $\tan \beta$. Note that $D_{s} \rightarrow \tau \nu_{\tau}$ does not provide any constraint in these examples.

\subsection{Flavour constraints for a CMSSM-like model}

We now consider the impact of the flavour constraints in the case of CMSSM with the addition of the non-renormalizable operators. In order to understand the implications on 
the BMSSM framework and, in particular, in order to allow for a simple comparison with CMSSM-like models, we investigate the following framework. The MSSM parameters that we use are those that would have corresponded to a CMSSM model specified by the five parameters: $m_{0}, m_{1 / 2}, A_{0}, \tan \beta$ and $\operatorname{sign}(\mu)$. Thus, the correlations between the low energy MSSM parameters are the same as those that would hold in a CMSSM framework. Let us emphasize again that one should not think about this set of parameters as coming from an extended CMSSM model, since the effects of the BMSSM physics at the few TeV scale on the running are not (and cannot be) taken into account.

In the scans, we took $-0.1<\epsilon_{1}<0$ and $\left|\epsilon_{2}\right|<0.1$, and varied the CMSSM parameters in the ranges $50<m_{0}<1200 \mathrm{GeV}, 100<m_{1 / 2}<1000 \mathrm{GeV},-1000<A_{0}<1000 \mathrm{GeV}$, $2<\tan \beta<50$ and imposed $\mu>0$ for $(g-2)_{\mu}$ compatibility, and generated about 400,000 random points.

Figure 5 shows in the $\tan \beta$ vs $m_{H^{+}}$plane a comparison of the allowed regions, and the effect of the different regions that are ruled out by the vacuum, $m_{h}$, and the flavour physics restrictions, using the same colour coding as in the previous section.

While in the MSSM case very low values for $\tan \beta$ are ruled out by the constraint on the mass of the lightest Higgs boson, in the BMSSM model for values of $m_{H^{+}} \gtrsim 300 \mathrm{GeV}$, this region is once again viable. Another important difference, is the effect of the $m_{h}$ constraint for the MSSM in region in which $2<\tan \beta<50$, and $150<m_{H^{+}}<500 \mathrm{GeV}$ which essentially rules out a large portion except for large values of $\tan \beta$, while in the BMSSM, it is mostly the vacuum constraint and $b \rightarrow s \gamma$ which exclude this area, in particular for the case with $\epsilon_{2}<0$.

The comparative effect for both models from the other flavour observables: $B \rightarrow \tau \nu$, $R_{l 23}, B \rightarrow D \ell \nu, D_{s} \rightarrow \tau \nu$ is rather mild. About $51 \%$ of the generated points are allowed in the case of $\epsilon_{2}>0,28 \%$ for $\epsilon_{2}<0$ and $21 \%$ for $\epsilon_{1}=\epsilon_{2}=0$.

\subsection{Flavour constraints for a NUHM-like model}

We explore in this section the parameter space of the non-universal Higgs mass (NUHM) framework [34], in which the universality assumptions of the soft SUSY breaking contributions to the Higgs masses are relaxed as compared to the CMSSM scenario. Within this framework, two additional free parameters, $m_{A}$ and $\mu$, add to the five universal parameters of the CMSSM scenario. Usually one trades $m_{A}$ for $m_{H^{+}}$through the mass relation, and therefore the charged Higgs boson mass can be treated essentially as a free parameter. This makes this model more attractive for the study of the Higgs sector. We also take into account the two extra parameters $\epsilon_{1}$ and $\epsilon_{2}$, in order to parametrize the effects of the non-renormalizable operators.

To study the effect of the different constraints on a NUHM-like scenario, we perform scans over $100<m_{0}<500 \mathrm{GeV}, 100<m_{1 / 2}<1000 \mathrm{GeV},-1000<A_{0}<1000 \mathrm{GeV}$, $100<m_{A}<1000 \mathrm{GeV}, 100<\mu<1000 \mathrm{GeV}$ and $2<\tan \beta<50$. In addition we scan over $-0.1<\epsilon_{1}<0$ and $\left|\epsilon_{2}\right|<0.1$. A total of about 400,000 random points are generated.

We present in figure 6 the allowed regions in the $\tan \beta$ vs $m_{H^{+}}$plane which satisfy the constraints from flavour observables and the effect of including the vacuum stability constraint for both $\epsilon_{2}>0$ (central panel) and $\epsilon_{2}<0$ (lower panel). For completeness we 

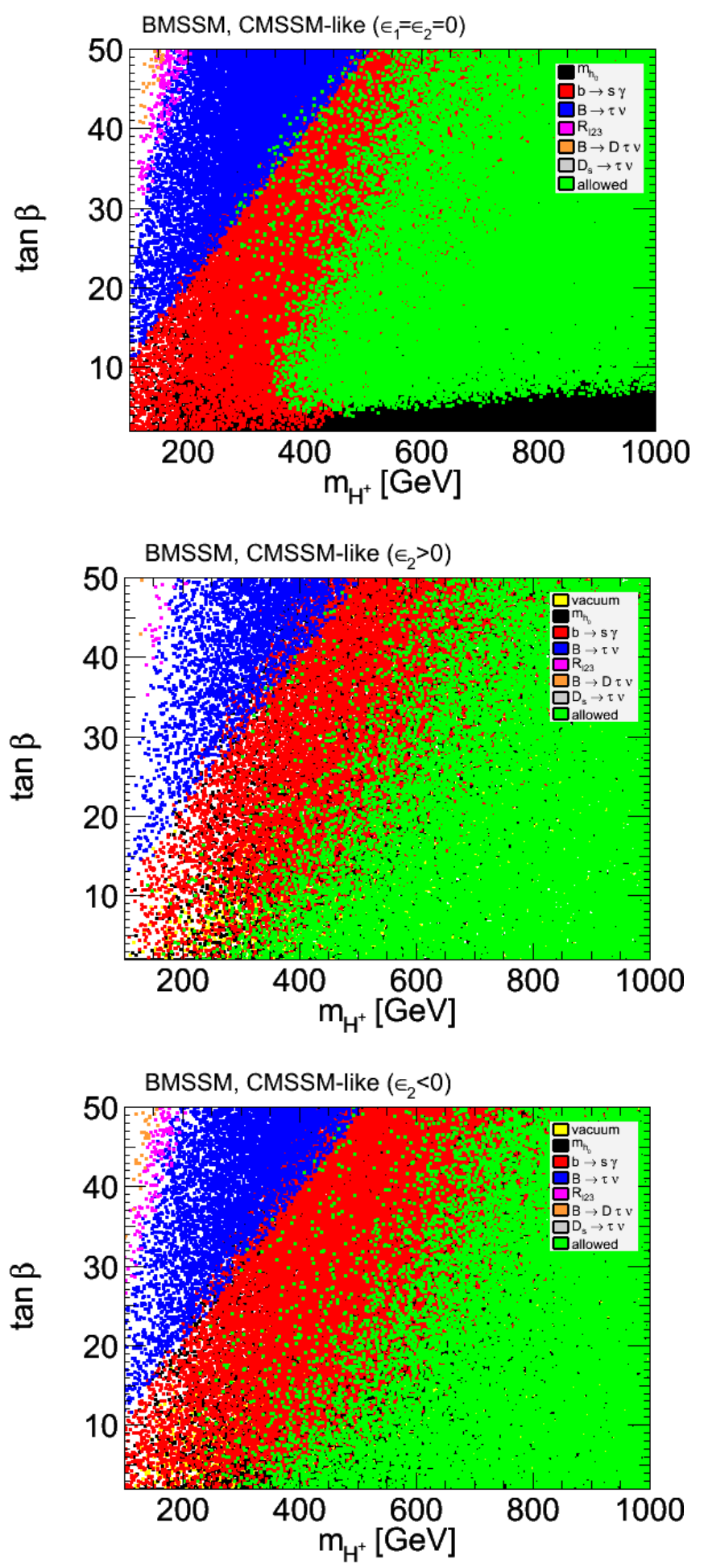

Figure 5. Flavour constraints on the $\left(m_{H^{+}}, \tan \beta\right)$ plane for the CMSSM (upper panel) and the CMSSM-like model with non-renormalizable operators with $\epsilon_{2}>0$ (central panel) and $\epsilon_{2}<0$ (lower panel). 

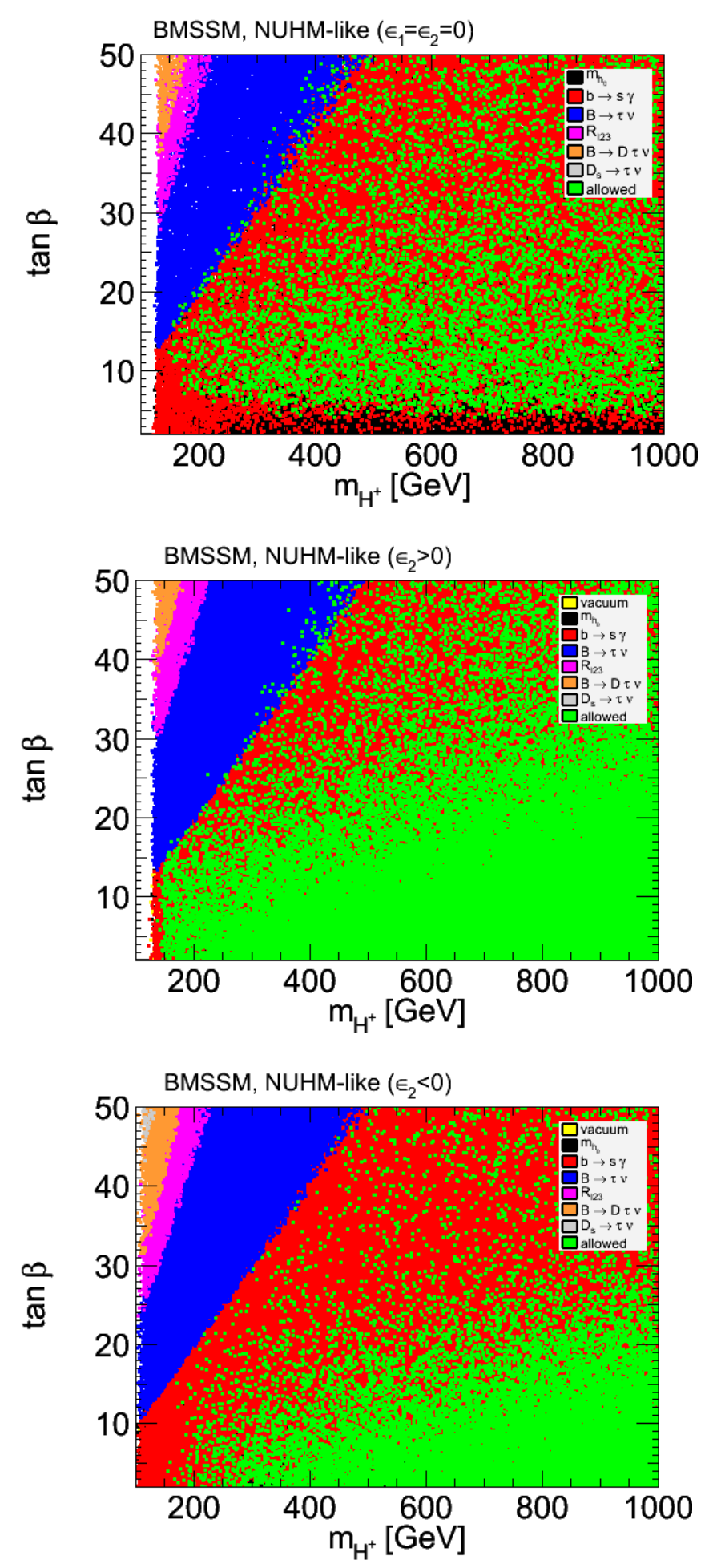

Figure 6. Flavour constraints on the $\left(m_{H^{+}}, \tan \beta\right)$ plane for the plain NUHM (upper panel) and the NUHM-like model with non-renormalizable operators with $\epsilon_{2}>0$ (central panel) and $\epsilon_{2}<0$ (lower panel). 
are also showing the plain NUHM case with $\epsilon_{1}=\epsilon_{2}=0$ in the upper panel. In the MSSM limit, very low values of $\tan \beta$ are excluded all across the range of values of $m_{H^{+}}$because of the Higgs mass; this region is allowed in the BMSSM for both positive and negative values of $\epsilon_{2}$. While the regions excluded from the constraint arising from $B \rightarrow \tau \nu_{\tau}$ are roughly similar for the MSSM and the BMSSM case with $\epsilon_{2}>0$, in the case of the BMSSM with $\epsilon_{2}<0$, the excluded region is somewhat enlarged reaching smaller values of $\tan \beta=10$ for small values of $m_{H^{+}}$. In this latter case a more preponderant role is played in excluding regions of parameter space by the constraints from $b \rightarrow s \gamma$ and the vacuum stability that rule out almost all the parameter space for $m_{H^{ \pm}} \lesssim 200 \mathrm{GeV}$. Regions with low values of $\tan \beta$ and low $m_{H^{+}}$are excluded. $14 \%$ of the generated points satisfy all the constraints for $\epsilon_{2}>0$ while $5 \%$ of the points are allowed for $\epsilon_{2}<0$ and $\epsilon_{1}=\epsilon_{2}=0$.

Figure 7 shows the impact of different flavour constraints in the $(\mu, \tan \beta)$ parameter space for $\epsilon_{2}>0$ (central panel) and $\epsilon_{2}<0$ (lower panel), and the standard NUHM case (upper panel). In this case, the bound on the Higgs boson mass excludes low values of $\tan \beta \lesssim 5$. For both BMSSM cases, the impact is lifted compared to the NUHM case. For negative $\epsilon_{2}$ values, the vacuum stability condition, $b \rightarrow s \gamma$ and also $B \rightarrow \tau \nu_{\tau}$ rule out a large portion of the parameter space corresponding to $\mu \gtrsim 700 \mathrm{GeV}$, compared to both the standard NUHM and the BMSSM with $\epsilon_{2}>0$.

\section{Conclusions}

We have presented a detailed analysis in the context of the BMSSM model of the effect of constraining the model from the perspective of flavour physics. In our study we have focused on the regions of parameter space in which foremost the vacuum is MSSM-like and sufficiently long-lived. We have then analyzed and compared the possible restrictions of the parameters in the model from flavour physics observables. We find that there can be significant differences compared to the MSSM case when we apply the flavour constraints.

In summary we find:

- The flavour constraints on EWBG, especially from $b \rightarrow s \gamma$ and $B \rightarrow \tau \nu_{\tau}$ limit the value of $\tan \beta \lesssim 15$ and furthermore for $\epsilon_{2}<0$, the vacuum stability constraint requires $\epsilon_{1} \lesssim-0.05$ and strongly restricts the allowed values of $\mu$ depending on the charged Higgs boson mass. For large enough values of the charged Higgs boson mass the constraint arising from $b \rightarrow s \gamma$ is determinant for intermediate values of $\tan \beta$.

- The flavour constraints in the model with a CMSSM parametrization show that very low values of $\tan \beta$ are allowed for large enough values of the charged Higgs boson mass. Here the exclusion of large regions in parameter space from the $b \rightarrow s \gamma$ constraint is further strengthened in particular when $\epsilon_{2}<0$.

- Finally in the model we studied with a NUHM-like parametrization, the $b \rightarrow s \gamma$ constraint has a stronger impact in excluding regions of parameter space especially for small values of the charged Higgs boson mass. In addition for $\epsilon_{2}<0$ values of $\mu \gtrsim 700 \mathrm{GeV}$ are ruled out by flavour constraints. 

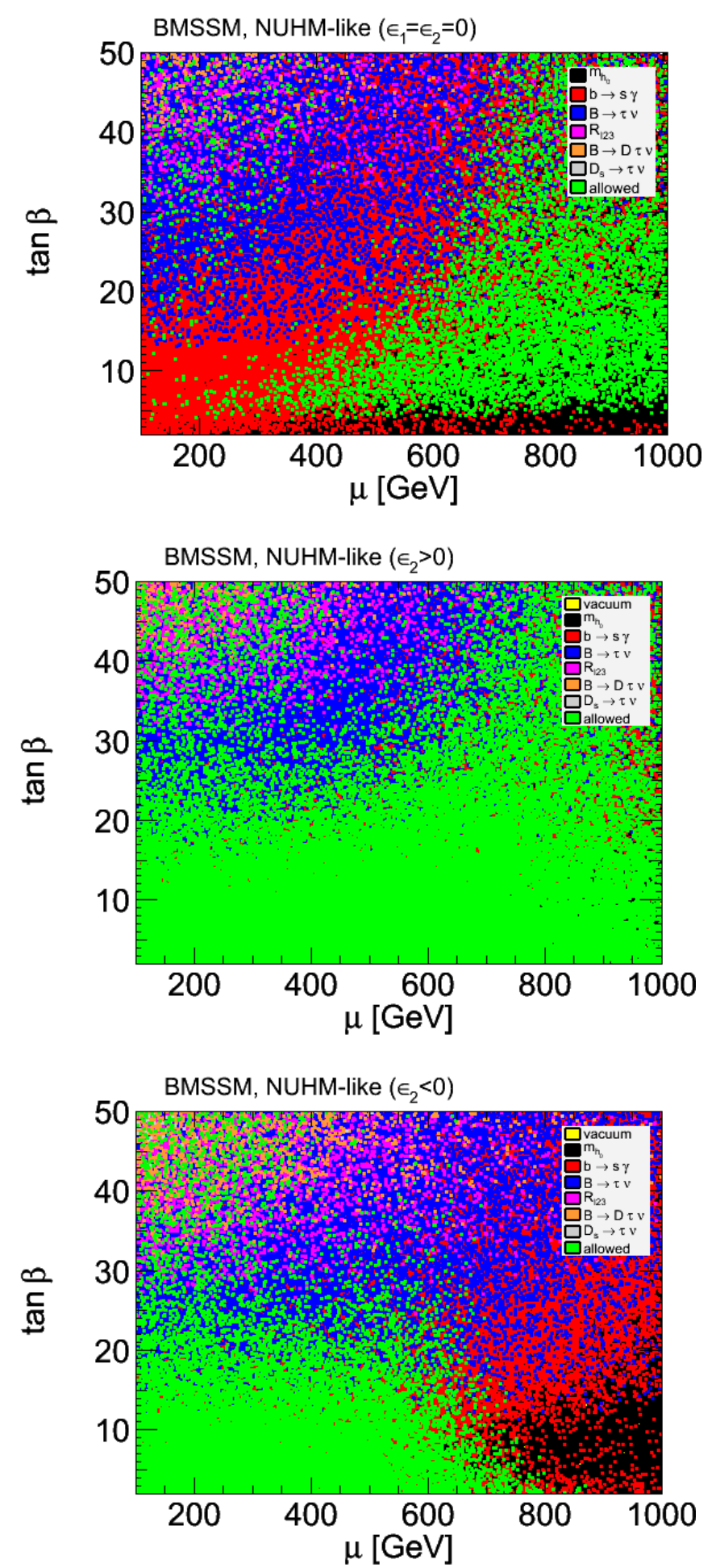

Figure 7. Flavour constraints on the $(\mu, \tan \beta)$ plane for the plain mSUGRA (upper panel) and the mSUGRA-like model with non-renormalizable operators with $\epsilon_{2}>0$ (central panel) and $\epsilon_{2}<0$ (lower panel). 


\section{Acknowledgments}

NB is supported by the DFG TRR33 'The Dark Universe'.

Open Access. This article is distributed under the terms of the Creative Commons Attribution Noncommercial License which permits any noncommercial use, distribution, and reproduction in any medium, provided the original author(s) and source are credited.

\section{References}

[1] A. Strumia, Bounds on Kaluza-Klein excitations of the SM vector bosons from electroweak tests, Phys. Lett. B 466 (1999) 107 [hep-ph/9906266] [SPIRES].

[2] J.A. Casas, J.R. Espinosa and I. Hidalgo, The MSSM fine tuning problem: a way out, JHEP 01 (2004) 008 [hep-ph/0310137] [SPIRES].

[3] A. Brignole, J.A. Casas, J.R. Espinosa and I. Navarro, Low-scale supersymmetry breaking: effective description, electroweak breaking and phenomenology, Nucl. Phys. B 666 (2003) 105 [hep-ph/0301121] [SPIRES].

[4] M. Pospelov, A. Ritz and Y. Santoso, Flavor and CP-violating physics from new supersymmetric thresholds, Phys. Rev. Lett. 96 (2006) 091801 [hep-ph/0510254] [SPIRES].

[5] M. Pospelov, A. Ritz and Y. Santoso, Sensitivity to new supersymmetric thresholds through flavour and CP-violating physics, Phys. Rev. D 74 (2006) 075006 [hep-ph/0608269] [SPIRES].

[6] M. Dine, N. Seiberg and S. Thomas, Higgs physics as a window beyond the MSSM (BMSSM), Phys. Rev. D 76 (2007) 095004 [arXiv:0707.0005] [SPIRES].

[7] I. Antoniadis, E. Dudas and D.M. Ghilencea, Supersymmetric models with higher dimensional operators, JHEP 03 (2008) 045 [arXiv: 0708.0383] [SPIRES].

[8] I. Antoniadis, E. Dudas, D.M. Ghilencea and P. Tziveloglou, MSSM with dimension-five operators (MSSM 5 ), Nucl. Phys. B 808 (2009) 155 [arXiv:0806.3778] [SPIRES].

[9] I. Antoniadis, E. Dudas, D.M. Ghilencea and P. Tziveloglou, Higher dimensional operators in the MSSM, AIP Conf. Proc. 1078 (2009) 175 [arXiv:0809.4598] [SPIRES].

[10] I. Antoniadis, E. Dudas, D.M. Ghilencea and P. Tziveloglou, MSSM Higgs with dimension-six operators, Nucl. Phys. B 831 (2010) 133 [arXiv:0910.1100] [SPIRES].

[11] I. Antoniadis, E. Dudas, D.M. Ghilencea and P. Tziveloglou, Beyond the MSSM Higgs with $D=6$ effective operators, Nucl. Phys. B 848 (2011) 1 [arXiv:1012.5310] [SPIRES].

[12] P. Batra and E. Ponton, Supersymmetric electroweak symmetry breaking, Phys. Rev. D 79 (2009) 035001 [arXiv:0809.3453] [SPIRES].

[13] M. Maniatis, The next-to-minimal supersymmetric extension of the standard model reviewed, Int. J. Mod. Phys. A 25 (2010) 3505 [arXiv:0906. 0777] [SPIRES].

[14] U. Ellwanger, C. Hugonie and A.M. Teixeira, The next-to-minimal supersymmetric standard model, Phys. Rept. 496 (2010) 1 [arXiv:0910.1785] [SPIRES].

[15] K. Blum and Y. Nir, Beyond MSSM baryogenesis, Phys. Rev. D 78 (2008) 035005 [arXiv: 0805.0097] [SPIRES]. 
[16] N. Bernal, K. Blum, Y. Nir and M. Losada, BMSSM implications for cosmology, JHEP 08 (2009) 053 [arXiv: 0906.4696] [SPIRES].

[17] K. Blum, C. Delaunay, M. Losada, Y. Nir and S. Tulin, CP violation beyond the MSSM: baryogenesis and electric dipole moments, JHEP 05 (2010) 101 [arXiv:1003.2447] [SPIRES].

[18] K. Cheung, S.Y. Choi and J. Song, Impact on the light higgsino-LSP scenario from physics beyond the minimal supersymmetric standard model, Phys. Lett. B 677 (2009) 54 [arXiv: 0903.3175] [SPIRES].

[19] M. Berg, J. Edsjo, P. Gondolo, E. Lundstrom and S. Sjors, Neutralino dark matter in BMSSM effective theory, JCAP 08 (2009) 035 [arXiv:0906.0583] [SPIRES].

[20] N. Bernal and A. Goudelis, Dark matter detection in the BMSSM, JCAP 03 (2010) 007 [arXiv: 0912.3905] [SPIRES].

[21] N. Bernal, Neutralino dark matter detection beyond the MSSM, arXiv:1005.2116 [SPIRES].

[22] K.J. Bae, H.D. Kim and S. Shin, Light neutralino dark matter with a very light Higgs for CoGeNT and DAMA/LIBRA data, Phys. Rev. D 82 (2010) 115014 [arXiv:1005.5131] [SPIRES].

[23] S. Shin, Light neutralino dark matter in light Higgs scenario related with the CoGeNT and DAMA/LIBRA results, arXiv:1011.6377 [SPIRES].

[24] S. Cassel and D.M. Ghilencea, A review of naturalness and dark matter prediction for the Higgs mass in MSSM and beyond, arXiv:1103.4793 [SPIRES].

[25] M. Carena, K. Kong, E. Ponton and J. Zurita, Supersymmetric Higgs bosons and beyond, Phys. Rev. D 81 (2010) 015001 [arXiv:0909.5434] [SPIRES].

[26] M. Carena, E. Ponton and J. Zurita, BMSSM Higgs bosons at the Tevatron and the LHC, Phys. Rev. D 82 (2010) 055025 [arXiv: 1005.4887] [SPIRES].

[27] M. Carena, E. Ponton and J. Zurita, SUSY Higgs bosons and beyond, PoS(DIS 2010) 212 [arXiv: 1006.5014] [SPIRES].

[28] P. Tziveloglou, Higher dimensional operators in the MSSM, Nucl. Phys. Proc. Suppl. 192-193 (2009) 190 [SPIRES].

[29] K.J. Bae, R. Dermisek, D. Kim, H.D. Kim and J.-H. Kim, Light Higgs scenario in BMSSM and LEP precision data, arXiv: 1001.0623 [SPIRES].

[30] A.H. Chamseddine, R.L. Arnowitt and P. Nath, Locally supersymmetric grand unification, Phys. Rev. Lett. 49 (1982) 970 [SPIRES].

[31] R. Barbieri, S. Ferrara and C.A. Savoy, Gauge models with spontaneously broken local supersymmetry, Phys. Lett. B 119 (1982) 343 [SPIRES].

[32] L.J. Hall, J.D. Lykken and S. Weinberg, Supergravity as the messenger of supersymmetry breaking, Phys. Rev. D 27 (1983) 2359 [SPIRES].

[33] N. Ohta, Grand unified theories based on local supersymmetry, Prog. Theor. Phys. 70 (1983) 542 [SPIRES].

[34] J.R. Ellis, K.A. Olive and Y. Santoso, The MSSM parameter space with non-universal Higgs masses, Phys. Lett. B 539 (2002) 107 [hep-ph/0204192] [SPIRES]. 
[35] K. Blum, C. Delaunay and Y. Hochberg, Vacuum (meta)stability beyond the MSSM, Phys. Rev. D 80 (2009) 075004 [arXiv:0905.1701] [SPIRES].

[36] M.S. Carena, A. Menon, R. Noriega-Papaqui, A. Szynkman and C.E.M. Wagner, Constraints on $B$ and Higgs physics in minimal low energy supersymmetric models, Phys. Rev. D 74 (2006) 015009 [hep-ph/0603106] [SPIRES].

[37] S. Heinemeyer, X. Miao, S. Su and G. Weiglein, B-physics observables and electroweak precision data in the CMSSM, $m G M S B$ and $m A M S B$, JHEP 08 (2008) 087 [arXiv:0805.2359] [SPIRES].

[38] D. Eriksson, F. Mahmoudi and O. Stal, Charged Higgs bosons in minimal supersymmetry: updated constraints and experimental prospects, JHEP 11 (2008) 035 [arXiv:0808.3551] [SPIRES].

[39] F. Mahmoudi, SuperIso: a program for calculating the isospin asymmetry of $B \rightarrow K^{*} \gamma$ in the MSSM, Comput. Phys. Commun. 178 (2008) 745 [arXiv:0710.2067] [SPIRES].

[40] F. Mahmoudi, SuperIso v2.3: a program for calculating flavor physics observables in supersymmetry, Comput. Phys. Commun. 180 (2009) 1579, http://superiso.in2p3.fr [arXiv: 0808.3144] [SPIRES].

[41] J.R. Ellis, S. Heinemeyer, K.A. Olive and G. Weiglein, Light heavy MSSM Higgs bosons at large $\tan \beta$, Phys. Lett. B 653 (2007) 292 [arXiv:0706. 0977] [SPIRES].

[42] F. Mahmoudi, New constraints on supersymmetric models from $b \rightarrow s \gamma$, JHEP 12 (2007) 026 [arXiv:0710.3791] [SPIRES].

[43] J.R. Ellis, S. Heinemeyer, K.A. Olive, A.M. Weber and G. Weiglein, The supersymmetric parameter space in light of B-physics observables and electroweak precision data, JHEP 08 (2007) 083 [arXiv:0706.0652] [SPIRES].

[44] Heavy Flavor Averaging Group collaboration, E. Barberio et al., Averages of b-hadron and c-hadron properties at the end of 2007, arXiv:0808.1297 [SPIRES].

[45] M. Misiak et al., The first estimate of $B(\bar{B} \rightarrow X / s \gamma)$ at $O\left(\alpha_{s}^{2}\right)$, Phys. Rev. Lett. 98 (2007) 022002 [hep-ph/0609232] [SPIRES].

[46] M. Misiak and M. Steinhauser, NNLO QCD corrections to the $B \rightarrow X_{s} \gamma$ matrix elements using interpolation in $m_{c}$, Nucl. Phys. B 764 (2007) 62 [hep-ph/0609241] [SPIRES].

[47] Particle Data Group collaboration, K. Nakamura et al., Review of particle physics, J. Phys. G 37 (2010) 075021 [SPIRES].

[48] W.-S. Hou, Enhanced charged Higgs boson effects in $B^{-} \rightarrow \tau$ anti- neutrino, $\mu$ anti-neutrino and $B \rightarrow \tau$ anti-neutrino $+X$, Phys. Rev. D 48 (1993) 2342 [SPIRES].

[49] A.G. Akeroyd and S. Recksiegel, The effect of $H^{ \pm}$on $B^{ \pm} \rightarrow \tau^{ \pm} \nu / \tau$ and $B^{ \pm} \rightarrow \mu^{ \pm} \nu_{\mu}, J$. Phys. G 29 (2003) 2311 [hep-ph/0306037] [SPIRES].

[50] B. Grzadkowski and W.-S. Hou, Solutions to the B meson semileptonic branching ratio puzzle within two Higgs doublet models, Phys. Lett. B 272 (1991) 383 [SPIRES].

[51] U. Nierste, S. Trine and S. Westhoff, Charged-Higgs effects in a new $B \rightarrow D \tau \nu$ differential decay distribution, Phys. Rev. D 78 (2008) 015006 [arXiv:0801.4938] [SPIRES].

[52] J.F. Kamenik and F. Mescia, $B \rightarrow D \tau \nu$ branching ratios: opportunity for lattice $Q C D$ and hadron colliders, Phys. Rev. D 78 (2008) 014003 [arXiv: 0802.3790] [SPIRES]. 
[53] BABAR collaboration, B. Aubert et al., Observation of the semileptonic decays $B \rightarrow$ $D^{*} \tau^{-} \bar{\nu}_{\tau}$ and evidence for $B \rightarrow D \tau^{-} \bar{\nu}_{\tau}$, Phys. Rev. Lett. 100 (2008) 021801 [arXiv:0709.1698] [SPIRES].

[54] J.L. Hewett, Searching for new physics with charm, hep-ph/9505246 [SPIRES].

[55] A.G. Akeroyd, Effect of $H^{ \pm}$on $D / s^{ \pm} \rightarrow \mu^{ \pm} \nu_{\mu}$ and $D / s^{ \pm} \rightarrow \tau^{ \pm} \nu_{\tau}$, Prog. Theor. Phys. 111 (2004) 295 [hep-ph/0308260] [SPIRES].

[56] A.G. Akeroyd and C.H. Chen, Effect of $H^{ \pm}$on $B^{ \pm} \rightarrow \tau^{ \pm} \nu_{\tau}$ and $D_{s}^{ \pm} \rightarrow \mu^{ \pm} \nu_{\mu}, \tau^{ \pm} \nu_{\tau}$, Phys. Rev. D 75 (2007) 075004 [hep-ph/0701078] [SPIRES].

[57] A.G. Akeroyd and F. Mahmoudi, Constraints on charged Higgs bosons from $D_{s}^{ \pm} \rightarrow \mu^{ \pm} \nu$ and $D_{s}^{ \pm} \rightarrow \tau^{ \pm} \nu$, JHEP 04 (2009) 121 [arXiv:0902.2393] [SPIRES].

[58] C.T.H. Davies et al., Update: precision $D_{s}$ decay constant from full lattice $Q C D$ using very fine lattices, Phys. Rev. D 82 (2010) 114504 [arXiv:1008.4018] [SPIRES].

[59] Flavianet Working Group on Kaon Decays collaboration, M. Antonelli et al., Precision tests of the Standard Model with leptonic and semileptonic kaon decays, arXiv:0801.1817 [SPIRES].

[60] HPQCD collaboration, E. Follana, C.T.H. Davies, G.P. Lepage and J. Shigemitsu, High precision determination of the $\pi, K, D$ and $D_{s}$ decay constants from lattice $Q C D$, Phys. Rev. Lett. 100 (2008) 062002 [arXiv:0706.1726] [SPIRES].

[61] M. Drees and K. Hagiwara, Supersymmetric contribution to the electroweak $\rho$ parameter, Phys. Rev. D 42 (1990) 1709 [SPIRES].

[62] M. Quirós, Electroweak baryogenesis and the Higgs and stop masses, Nucl. Phys. Proc. Suppl. 101 (2001) 401 [hep-ph/0101230] [SPIRES].

[63] M. Carena, G. Nardini, M. Quirós and C.E.M. Wagner, The baryogenesis window in the MSSM, Nucl. Phys. B 812 (2009) 243 [arXiv:0809.3760] [SPIRES].

[64] J.R. Espinosa, Dominant two-loop corrections to the MSSM finite temperature effective potential, Nucl. Phys. B 475 (1996) 273 [hep-ph/9604320] [SPIRES].

[65] M.S. Carena, M. Quirós and C.E.M. Wagner, Opening the window for electroweak baryogenesis, Phys. Lett. B 380 (1996) 81 [hep-ph/9603420] [SPIRES].

[66] A. Brignole, J.R. Espinosa, M. Quirós and F. Zwirner, Aspects of the electroweak phase transition in the minimal supersymmetric standard model, Phys. Lett. B 324 (1994) 181 [hep-ph/9312296] [SPIRES].

[67] J.R. Espinosa, M. Quirós and F. Zwirner, On the electroweak phase transition in the minimal supersymmetric standard model, Phys. Lett. B 307 (1993) 106 [hep-ph/9303317] [SPIRES].

[68] J.R. Espinosa, Dominant two-loop corrections to the MSSM finite temperature effective potential, Nucl. Phys. B 475 (1996) 273 [hep-ph/9604320] [SPIRES].

[69] M.S. Carena, M. Quirós and C.E.M. Wagner, Opening the window for electroweak baryogenesis, Phys. Lett. B 380 (1996) 81 [hep-ph/9603420] [SPIRES].

[70] D. Delepine, J.M. Gerard, R. Gonzalez Felipe and J. Weyers, A light stop and electroweak baryogenesis, Phys. Lett. B 386 (1996) 183 [hep-ph/9604440] [SPIRES].

[71] J.M. Cline and K. Kainulainen, Supersymmetric electroweak phase transition: beyond perturbation theory, Nucl. Phys. B 482 (1996) 73 [hep-ph/9605235] [SPIRES]. 
[72] M. Losada, High temperature dimensional reduction of the MSSM and other multi-scalar models, Phys. Rev. D 56 (1997) 2893 [hep-ph/9605266] [SPIRES].

[73] M. Laine, Effective theories of MSSM at high temperature, Nucl. Phys. B 481 (1996) 43 [Erratum ibid. B 548 (1999) 637] [hep-ph/9605283] [SPIRES].

[74] G.R. Farrar and M. Losada, SUSY and the electroweak phase transition, Phys. Lett. B 406 (1997) 60 [hep-ph/9612346] [SPIRES].

[75] D. Bödeker, P. John, M. Laine and M.G. Schmidt, The 2-loop MSSM finite temperature effective potential with stop condensation, Nucl. Phys. B 497 (1997) 387 [hep-ph/9612364] [SPIRES].

[76] B. de Carlos and J.R. Espinosa, The baryogenesis window in the MSSM, Nucl. Phys. B 503 (1997) 24 [hep-ph/9703212] [SPIRES].

[77] M. Laine and K. Rummukainen, The MSSM electroweak phase transition on the lattice, Nucl. Phys. B 535 (1998) 423 [hep-lat/9804019] [SPIRES].

[78] M.S. Carena, M. Quirós and C.E.M. Wagner, Electroweak baryogenesis and Higgs and stop searches at LEP and the Tevatron, Nucl. Phys. B 524 (1998) 3 [hep-ph/9710401] [SPIRES].

[79] M. Losada, The two-loop finite-temperature effective potential of the MSSM and baryogenesis, Nucl. Phys. B 537 (1999) 3 [hep-ph/9806519] [SPIRES].

[80] M. Laine and K. Rummukainen, Higgs sector CP-violation at the electroweak phase transition, Nucl. Phys. B 545 (1999) 141 [hep-ph/9811369] [SPIRES].

[81] M. Losada, Mixing effects in the finite-temperature effective potential of the MSSM with a light stop, Nucl. Phys. B 569 (2000) 125 [hep-ph/9905441] [SPIRES].

[82] M. Laine and M. Losada, Two-loop dimensional reduction and effective potential without temperature expansions, Nucl. Phys. B 582 (2000) 277 [hep-ph/0003111] [SPIRES].

[83] V. Cirigliano, Y. Li, S. Profumo and M.J. Ramsey-Musolf, MSSM baryogenesis and electric dipole moments: an update on the phenomenology, JHEP 01 (2010) 002 [arXiv:0910.4589] [SPIRES].

[84] Y. Li, S. Profumo and M. Ramsey-Musolf, Bino-driven electroweak baryogenesis with highly suppressed electric dipole moments, Phys. Lett. B 673 (2009) 95 [arXiv:0811.1987] [SPIRES].

[85] A. Djouadi, J.-L. Kneur and G. Moultaka, SuSpect: a Fortran code for the supersymmetric and Higgs particle spectrum in the MSSM, Comput. Phys. Commun. 176 (2007) 426 [hep-ph/0211331] [SPIRES].

[86] CDF AND D0 collaboration and others, Combination of CDF and D0 results on the mass of the top quark, arXiv:1007.3178 [SPIRES]. 